\title{
A local approach to assess effects of specimen geometry on cleavage fracture toughness in reactor pressure vessel steels
}

DOI:

10.1115/pvp2018-85063

\section{Document Version}

Accepted author manuscript

Link to publication record in Manchester Research Explorer

Citation for published version (APA):

Sarzosa, D., Savioli, R., Ruggieri, C., Jivkov, A., \& Beswick, J. (2018). A local approach to assess effects of specimen geometry on cleavage fracture toughness in reactor pressure vessel steels. In Proceedings of the ASME 2018 Pressure Vessels and Piping Conference [PVP2018-85063] American Society of Mechanical Engineers. https://doi.org/10.1115/pvp2018-85063

\section{Published in:}

Proceedings of the ASME 2018 Pressure Vessels and Piping Conference

\section{Citing this paper}

Please note that where the full-text provided on Manchester Research Explorer is the Author Accepted Manuscript or Proof version this may differ from the final Published version. If citing, it is advised that you check and use the publisher's definitive version.

\section{General rights}

Copyright and moral rights for the publications made accessible in the Research Explorer are retained by the authors and/or other copyright owners and it is a condition of accessing publications that users recognise and abide by the legal requirements associated with these rights.

\section{Takedown policy}

If you believe that this document breaches copyright please refer to the University of Manchester's Takedown Procedures [http://man.ac.uk/04Y6Bo] or contact uml.scholarlycommunications@manchester.ac.uk providing relevant details, so we can investigate your claim.

\section{OPEN ACCESS}




\section{DRAFT \\ A LOCAL APPROACH TO ASSESS EFFECTS OF SPECIMEN GEOMETRY ON CLEAVAGE FRACTURE TOUGHNESS IN REACTOR PRESSURE VESSEL STEELS}

\author{
Diego F. B. Sarzosa, Rafael G. Savioli, Claudio Ruggieri \\ Department of Naval Architecture \\ and Ocean Engineering \\ University of São Paulo, São Paulo, Brazil \\ Email:dsarzosa@usp.br \\ rafaelgsavioli@gmail.com \\ claudio.ruggieri@usp.br
}

\author{
Andrey P. Jivkov, Jack Beswick \\ School of Mechanical, Aerospace, \\ and Civil Engineering \\ University of Manchester, Manchester, UK \\ Email:Andrey.Jivkov@manchester.ac.uk \\ jack.beswick@postgrad.manchester.ac.uk
}

\begin{abstract}
This work presents recent improvements in the micromechanical failure citeria based on the Weibull stress $\left(\sigma_{w}\right)$ concept for prediction of cleavage fracture in ferritic steels. The model is applied in SE(B) specimens extracted from an ASTM A533 pressure vessel steel having different levels of stress triaxiality at the crack tip. Nonlinear 3D finite element models with dimensions matching the tested specimens were built to provide the necessary crack tip stresses at the fracture process zone for calculation of $\sigma_{w^{-}} J$ evolution from wich the variation of characteristic toughness value $\left(J_{0}\right)$ between different cracked geometries can be estimated. The application of this methodology for the material used at this study is able to predict $J_{0}$ for $\mathrm{SE}(\mathrm{B})$ specimens with very shallow crack size ratio $a / W=0.05$, short crack $a / W=0.2$ and deep crack $a / W=0.4$. The reported fracture toughness values, for specimens having very shallow crack size ratio, is an additional contribution of this study.
\end{abstract}

\section{INTRODUCTION}

Fracture testing of ferritic structural steels in the ductileto-brittle (DBT) transition region consistently reveals a significant effect of specimen geometry on cleavage toughness values as measured by the $J$-integral at cleavage instability, $J_{c}$, and its equivalent toughness measure $K_{J C}$ (see [1],[2] for illustrative data). These studies show significant elevations in the elastic- plastic fracture toughness for shallow crack bend specimens and tension loaded geometries of ferritic steels tested in the transition region, where transgranular cleavage triggers macroscopic fracture. At increased loads in a cracked specimen or structure, the initially strong SSY fields gradually change to fields under large scale yielding (LSY) as crack-tip plastic zones increasingly merge with the global bending plasticity on the nearby traction free boundaries. This phenomenon, often termed loss of constraint, contributes to the apparent increased toughness of shallow cracked and tension loaded geometries observed in fracture testing. Once SSY conditions no longer apply, larger $J$-values in the finite body are necessary to generate a highly stressed region ahead of crack tip sufficient to trigger cleavage. These features have enormous practical implications in defect assessment procedures, particularly repair decisions and life extension programs of in-service structures as well as structural design specifications. Moreover, cleavage fracture is a highly localized phenomenon which exhibits strong sensitivity to material characteristics at the microlevel. In particular, the random inhomogeneity in local features of the material causes large scatter in measured values of cleavage fracture toughness. The coupled effects of constraint loss and inherent scatter of toughness values in the DBT region have enormous practical implications in accurate assessments of structural integrity and greatly complicate the development of defect assessment procedures, particularly repair decisions and life extension programs of in-service structures as well as structural 
design specifications.

To address this issue, advanced methodologies for cleavage fracture assessments endeavor to describe the fracture process based on local failure criteria that relate the local fracture conditions with macroscopic (global) fracture parameters and to the subsequent prediction of constraint variations on cleavage fracture toughness. Such methodologies are most often referred to as local approaches $[3,4,5]$ and are essentially based on probabilistic models incorporating weakest link statistics to describe material failure caused by transgranular cleavage for a wide range of loading conditions and crack configurations.

In particular, the work of Beremin [3] provides the basis for establishing a relationship between the microregime of fracture and macroscopic crack driving forces (such as the $J$-integral) by introducing the Weibull stress $\left(\sigma_{w}\right)$ as a probabilistic fracture parameter. In the context of probabilistic fracture mechanics, the Weibull stress emerges as a near-tip fracture parameter to describe the coupling of remote loading with a micromechanics model which incorporates the statistics of microcracks (weakest link philosophy). A key feature of this methodology is that $\sigma_{w}$ incorporates both the effects of stressed volume (the fra cture process zone) and the potentially strong changes in the character of the near-tip stress fields due to constraint loss which thus provides the necessary framework to correlate fracture toughness for varying crack configurations under different loading (and possibly temperature) conditions.

One of the main theoretical objections to the Weibull stress concept is that the probabilistic framework from which it is derived largely relies on the assumption that Griffith-like microcracks form immediately upon the onset of yielding and thus the associated statistical distribution of microcrack size remains unchanged with increased loading and deformation. A substantial number of experimental studies $[6,7,8,9,10]$ indicates the potential effects of plastic strain on cleavage microcracking in ferritic steels at varying temperatures which can lead to a marked influence on the density of Griffith-like microcracks directly connected to the material fracture behavior at the microscale. Since any cleavage fracture model incorporating the statistics of microcracks (weakest link philosophy), such as the $\sigma_{w}$-based methodology, involves a local Griffith instability of the largest of most favorably oriented microcrack, it becomes clear that varying plastic strains conditions in the near-tip region correlate directly with varying likelihood of cleavage failure.

This work describes a micromechanics methodology based upon a local failure criterion to predict the effects of crack tip triaxiality and plastic strain on fracture toughness values. This study is part of a collaborative program between University of São Paulo and University of Manchester. An intensive experimental campaign was carried out to obtain the necessary fracture toughness data for an A533 Gr.B reactor pressure vessel steel under different stress conditions. The chosen material was investigated under four different initial conditions: (a) as received; (b)
$5 \%$ of plastic strain; (c) residual stresses and (d) plastic strain and residual stresses. The results reported here correspond to the as received conditions. The central objective of this collaboration is to explore and further extend application of a micromechanics model incorporating the influence of plastic strain on cleavage fracture developed in previous work by Ruggieri and Dodds [11] to correct fracture toughness for effects of geometry and constraint loss. These experimental results provide the cleavage fracture resistance data needed to assess specimen geometry and plastic strain effects on experimentally measured $J_{c}$-values.

Very detailed non-linear finite element analyses for 3-D models of plane-sided fracture specimens with different crack sizes provide the evolution of near-tip stress field with the increased of macroscopic loading (in terms of the $J$-integral) to define the relationship between $\tilde{\sigma}_{w}$ and $J$ from which the variation of fracture toughness across different crack configurations is predicted. For the tested material in the as received condition, the standard Weibull stress methodology effectively removes the geometry dependence on $J_{c}$-values and predicts accurately the characteristic toughness value for specimen having different crack sizes. The application of the modified Weibull stress approach, incorporating the plastic strain correction, to predict the effects of initial plastic strain and initial residual stresses will be reported in a future article which is under development.

\section{LOCAL APPROACH FOR CLEAVAGE FRACTURE}

This section introduces the essential features of the modified Weibull stress framework needed to unify toughness measures across different crack configurations and loading modes. The brief description that follows draws heavily on the recent work of Ruggieri and Dodds (R\&D) [11, 12] while, at the same time, providing the basis to assess the coupling effects of specimen geometry and plastic strain on cleavage fracture toughness predictions.

\section{The Modified Weibull Stress}

Development of a micromechanics model for cleavage fracture incorporating effects of plastic strain begins by assuming the fracture process zone (FPZ) in a stressed cracked body illustrated in Fig. 1(a) in which a small volume element, $\delta V$, is subjected to the principal stress, $\sigma_{1}$, and associated effective plastic strain, $\varepsilon_{p}$. Here, only microcracks formed from the cracking of brittle particles, such as carbides, in the course of plastic deformation contribute to cleavage fracture and, further, the fraction of fractured particles increases with increased matrix plastic strain [13].

An approximate account of such a micromechanism can be made by considering that a fraction, $\Psi_{c}$, of the total number of brittle particles in the FPZ nucleates the microcracks which are eligible to propagate unstably and, further, that $\Psi_{c}$ is a function of plastic strain but plausibly independent of microcrack size as 
pictured in Fig. 1(b). This approximation simplifies the treatment of the elemental failure probability associated with $\delta V$ to arrive at a closed form for the failure probability of the stressed cracked body while, at the same time, retaining strong contact with the Weibull stress concept introduced by Beremin [3].

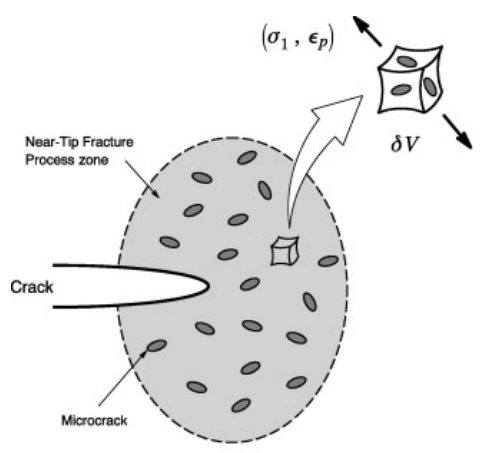

(a)

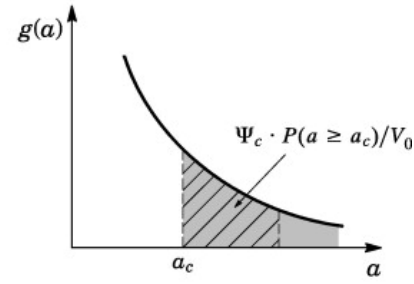

(b)
FIGURE 1. (a) Near tip fracture process zone ahead of macroscopic crack having randomly distributed flaws. (b) Schematic of power-law type microcrack size distribution.

Following standard procedures based on the weakest link approach (see R\&D [11]), a limiting distribution for the cleavage fracture stress can expressed as a two-parameter Weibull function [14] in the form

$$
P_{f}\left(\sigma_{1}, \varepsilon_{p}\right)=1-\exp \left[-\frac{1}{V_{0}} \int_{\Omega} \Psi_{c}\left(\varepsilon_{p}\right) \cdot\left(\frac{\sigma_{1}}{\sigma_{u}}\right)^{m} d \Omega\right]
$$

where $\Omega$ is the volume of the near-tip fracture process zone most often defined as the loci where $\sigma_{1} \geq \lambda \sigma_{y s}$, with $\sigma_{y s}$ denoting the material yield stress and $\lambda \geq 1$, and $V_{0}$ represents a reference volume. Parameters $m$ and $\sigma_{u}$ appearing in Eq. (1) denote the Weibull modulus and the scale parameter of the Weibull distribution. In particular, $m$ defines the shape of the probability density function describing the microcrack size, $a$, which is of the form $g(a) \propto a^{-\zeta}$ with $m=2 \zeta-2$. Moreover, since the reference volume, $V_{0}$, only scales $g(a)$ but does not change the distribution shape, it has no effect on $m$ and is conveniently assigned a unit value in computations. The above integral evaluated over $\Omega$ contains two contributions: one is from the principal stress criterion for cleavage fracture characterized in terms of $\sigma_{1}$ and the other is due the effective plastic strain, $\varepsilon_{p}$, which defines the number of eligible Griffith-like microcracks nucleated from the brittle particles effectively controlling cleavage fracture. Similar to the Beremin model [3], a simple manipulation of Eq. (1) then motivates the notion of a modified Weibull stress, $\tilde{\sigma}_{w}$, defined by

$$
\tilde{\sigma}_{w}=\left[\frac{1}{V_{0}} \int_{\Omega} \Psi_{c}\left(\varepsilon_{p}\right) \cdot \sigma_{1}^{m} d \Omega\right]^{1 / m}
$$

where it is noted that setting $\Psi_{c}=1$ recovers the standard Beremin model.

To arrive at a simpler form for the failure probability of cleavage fracture including effects of plastic strain, we follow similar arguments to those given by $R \& D$ [11] to define the fraction of fractured particles as a two-parameter Weibull distribution given by Wallin and Laukkanen [15] as

$$
\Psi_{c}=1-\exp \left[-\left(\frac{\sigma_{p f}}{\sigma_{p r s}}\right)^{\alpha_{p}}\right]
$$

where $\sigma_{p r s}$ is the particle reference fracture stress, $\alpha_{p}$ denotes the Weibull modulus of the particle fracture stress distribution and $\sigma_{p f}=\sqrt{1.3 \sigma_{1} \varepsilon_{p} E_{d}}$ characterizes the particle fracture stress in which $\sigma_{1}$ is the maximum principal stress, $\varepsilon_{p}$ denotes the effective matrix plastic strain and $E_{d}$ represents the particle's elastic modulus. Here, it is understood that the particle reference stress, $\sigma_{p r s}$, represents an approximate average for the distribution of the particle fracture stress.

Now, substitution of Eq. (3) into (2) then provides a modified Weibull stress incorporating a simplified distribution for the fractured particle in the form

$$
\tilde{\sigma}_{w}=\left[\frac{1}{V_{0}} \int_{\Omega}\left\{1-\exp \left[-\left(\frac{\sigma_{p f}}{\sigma_{p r s}}\right)^{\alpha_{p}}\right]\right\} \cdot \sigma_{1}^{m} d \Omega\right]^{1 / m}
$$

in which the effect of plastic strain on cleavage fracture probability enters into $\tilde{\sigma}_{w}$ through the particle fracture stress, $\sigma_{p f}$. The modified Weibull stress thus emerges as a crack-front parameter to couple remote loading with a micromechanics model which incorporates the statistics of microcracks and plastic strain effects. Unstable crack propagation (cleavage) occurs at a critical value of $\tilde{\sigma}_{w}$. Under increased remote loading described by $J$ (or, equivalently $K_{J}$ or CTOD), differences in evolution of the modified Weibull stress, $\tilde{\sigma}_{w}$, reflect the potentially strong variations in crack-front stress and strain fields due to the effects of constraint loss. The inherently 3-D formulation for $\tilde{\sigma}_{w}$ defined by Eq. (4) readily accommodates variations in $J$ along the crack front and the effects of shallow crack vs. deep cracks or large cross section vs. subsize cross section configurations. A toughness constraint correction procedure then derives from ratios of $J$-values in the test specimens with different geometries at identical values of $\tilde{\sigma}_{w}$ 
as addressed later.

Calibration of the Modified Weibull Stress Parameters

Calibration of the Weibull stress parameters appearing in previous Eq. (4) is a key step in the multiscale procedure to correct fracture toughness for effects of geometry and constraint loss adopted in the present analysis. Specifically, the Weibull modulus, $m$, plays a major role in the process to correlate toughness values across different crack configurations as this parameter alters the $\tilde{\sigma}_{w}$ vs. $J$ trajectories needed to correlate the Weibull stress at fracture, denoted $\tilde{\sigma}_{w, c}$. Similar arguments also apply to the parameters defining the fraction, $\Psi_{c}$, of the microcracks which are eligible to propagate unstably. Because the Weibull modulus, $m$, characterizes the distribution of Griffith-like microcracks associated with the cleavage fracture process (see Ruggieri and Dodds $[11,12]$ and references therein), we can advantageously evaluate $m$ and $\Psi_{c}$ using a two-step process derived from fracture toughness testing in combination with detailed finite element analyses as illustrated in Fig. 2.

First, parameter $m$ is determined to establish the best correction for cleavage fracture toughness data measured from two sets of test specimens exhibiting widely different toughness behavior based on the standard Beremin model (i.e., $\Psi_{c}=1$ ). The procedure essentially relies on the toughness scaling model (TSM) proposed earlier by Ruggieri and Dodds [16] building upon the interpretation of $\tilde{\sigma}_{w}$ as the (probabilistic) crack tip driving force coupled with the condition that cleavage fracture occurs when $\tilde{\sigma}_{w}$ reaches a critical value, $\tilde{\sigma}_{w, c}$. For the same material at temperatures within the DBT region and sufficiently close to the test temperature, the scaling model requires the attainment of a specified value for $\tilde{\sigma}_{w}$ to trigger cleavage fracture across different crack configurations even though the loading parameter (measured here by the $J$-integral) may vary widely due to constraint loss. Once the relation between the modified Weibull stress $\left(\tilde{\sigma}_{w}\right)$ and applied loading $(J)$ for a given value of the Weibull modulus, $m$, is determined, the calibration scheme adopted here defines the calibrated Weibull modulus for the material as the $m$-value, denoted $m_{0}$, that corrects the characteristic toughness $J_{0}^{B}$ corresponding to a low constraint configuration (denoted as configuration B) to its equivalent $J_{0}^{A}$ corresponding to a high constraint configuration (denoted as configuration $\mathbf{A}$ ) such that the residual toughness values defined as $R(m)=\left(J_{0, m}^{A}-J_{0}^{A}\right) / J_{0}^{A}$ is minimized. Gao et al. [17] and Ruggieri [18] discuss additional details of the calibration process for the Weibull modulus.

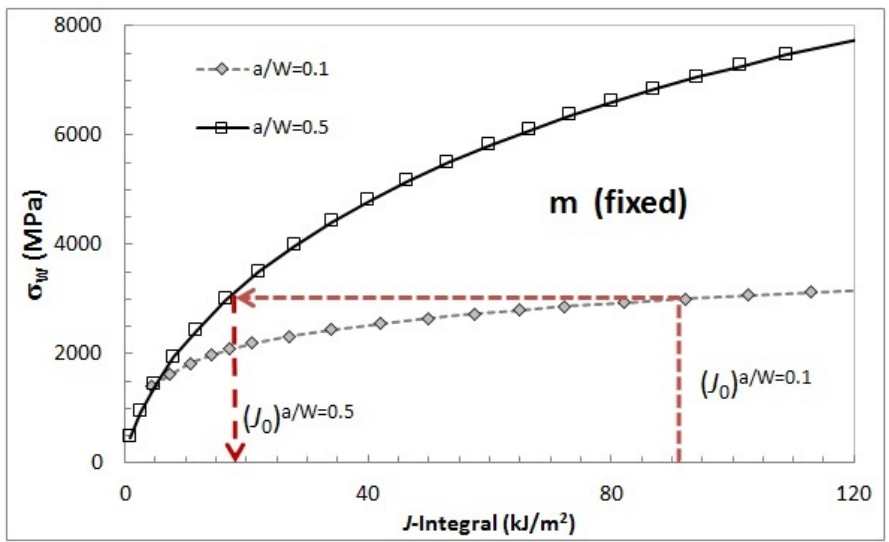

FIGURE 2. Calibration of Weibull shape parameter using the very shallow crack and the deep crack Three-point bend specimes.

With the Weibull modulus (and, presumably, the microcrack distribution) thus determined and now assumed fixed throughout the analysis, the calibration process then proceeds by evaluation of the function $\Psi_{c}$ that again provides the best correction for cleavage fracture toughness data measured from the two sets of test specimens utilized at the onset of the calibration procedure using the toughness scaling model. Here, the $\tilde{\sigma}_{w}-J$ relations for a fixed $m_{0}$-value enable determination of a $\Psi_{c}$ that provides the best correction $J_{0}^{B} \rightarrow J_{0}^{A}$ such that the residual, $R\left(\Psi_{c}\right)$, is minimized. The calibrated values for $m$ and $\Psi_{c}$ clearly do not constitute a unique pair of parameters; for example, a slightly different $m$-value may be compensated for by a different $\Psi_{c}$.

Nevertheless, the procedure is relatively simple and, perhaps most importantly, does preserve the character of parameter $m$ in describing the microcrack distribution. Moreover, it is well to keep in mind that, within the present context, the calibrated Weibull stress parameters, $m$ and $\Psi_{c}$, are only loosely connected to the actual microcrack distribution. Because of the nature of the calibration process adopted here, which relies on the TSM and macroscopic measures of fracture toughness, $m$ and $\Psi_{c}$ should be interpreted as phenomenological parameters that bring fracture toughness predictions into agreement, rather than accurate descriptors of the metallurgical features. Further investigation and sensitivity analysis related to the two-step parameter calibration of the modified Weibull stress approach and implications for fracture toughness predictions is in progress and will be presented in a forthcoming publication.

\section{EXPERIMENTAL DETAILS}

To investigate the effects of specimen geometry on fracture toughness values, a series of toughness tests was conducted on three-point bend $\mathrm{SE}(\mathrm{B})$ fracture specimens with varying crack sizes. In-plane dimensions of the specimen are shown in Fig.3. 
Adopted dimensions are $B=25 \mathrm{~mm}, W=50 \mathrm{~mm}, S=4 W$ with $a / W=0.05, a / W=0.2$ and $a / W=0.5$. Here, $a$ is the crack size, $W$ denotes the specimen width, $B$ represents the specimen thickness and $S$ is the load span. BS7448 standard [19] provides additional details for the geometry and dimensions of the tested fracture specimens.

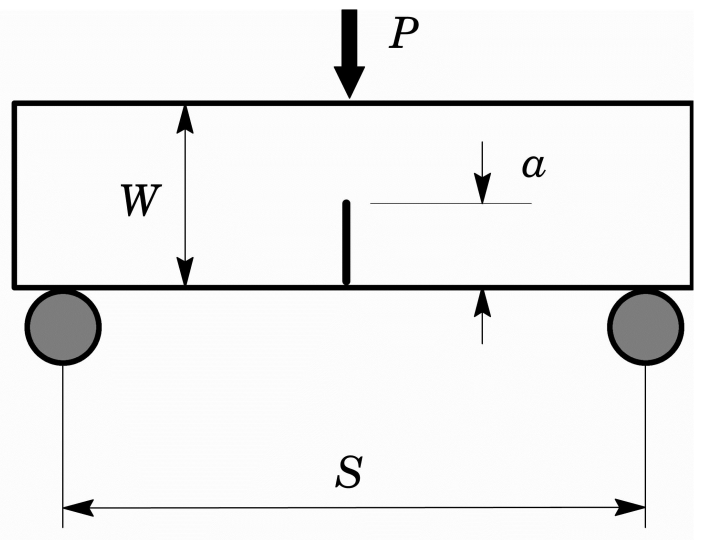

FIGURE 3. Specimen geometries used in fracture tests on the A533 steel: Three-point bend specimen specimen.

\section{Tensile Tests}

Mechanical tensile tests were conducted on standard tensile specimens. These tests provide the room temperature $\left(T=20^{\circ} \mathrm{C}\right.$ C) stress-strain data and tensile properties at the same temperature at wchich the fracture toughness tests were performed. Fig.3 displays the measured engineering tensile stress-strain properties for the A533 steel. The tensile yield strength is $\sigma_{y s}=530$ $\mathrm{MPa}$ and the ultimate tensile strength is $\sigma_{u t s}=660 \mathrm{MPa}$ at room temperature $\left(20^{\circ} \mathrm{C}\right)$. At "cryogenic" temperature $\left(-140^{\circ} \mathrm{C}\right)$, the material has $720 \mathrm{MPa}$ yield stress $\left(\sigma_{y s}\right)$ and $880 \mathrm{MPa}$ tensile strength $\left(\sigma_{u t s}\right)$. Additional material properties include Young's modulus $E=205 \mathrm{GPa}$ and Poisson's ratio $v=0.3$ at room temperature and $E=215 \mathrm{GPa}$ at $T=-140^{\circ} \mathrm{C}$.

\section{Fracture Toughness Tests}

A series of fracture toughness tests were conducted on the single edge bend $\mathrm{SE}(\mathrm{B})$ specimens in three-point loading. The tests were carried out at $T=-140^{\circ} \mathrm{C}$, to simulate radiation embrittlement that may be seen in RPV steels during service, and to also ensure as far as possible a cleavage mode of failure.

The test specimens have been machined following BS7448 standard [19]. After machining the specimens a fatigue precrack was introduced at the notch root by cyclic loading the specimens under three-pointbending at room temperature in accordance with the stringent requirements of [19] .

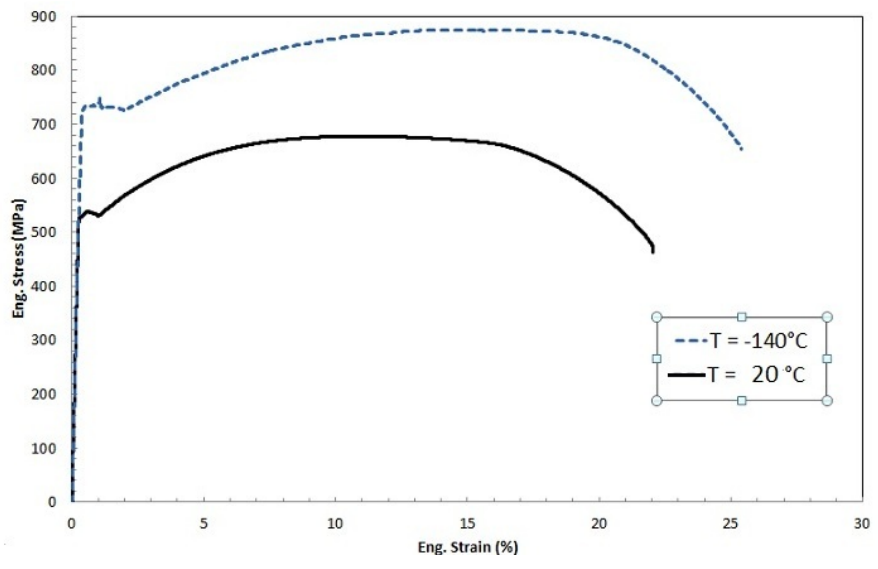

FIGURE 4. Engineering tensile stress-strain properties for the A533 steel.

All fracture toughness testing was carried out at Amec Foster Wheeler's Newton house site, on a Schenk $250 \mathrm{kN}$ testing machine. The tests were carried out following all necessary subsequent pre-processes. All tests were carried out to, as far as possible comply with the relevant standards $[19,20]$, despite the non-standard geometry with $a / W=0.05$. Records of load vs. crack mouth opening displacements (CMOD) were obtained for each specimen using a clip gauge mounted on an integrated knife-edge machined into the notch mouth. The specimens are cooled using liquid nitrogen in an environmental chamber, with the temperature monitored by a thermocouple, which is installed in contact with the specimen. To ensure that the entire specimen has cooled to the appropriate temperature, the cooling continues after the temperature reaches $T=-140^{\circ} \mathrm{C}$ for $1 \mathrm{~min} / \mathrm{mm}$ thickness of the specimen. Once the specimen is sufficiently cooled the test is carried out.

Following testing crack growth is measured. This involves regarding the crack front under a microscope and measuring how far the crack has extended across the thickness of the specimen. This is easily discernible through a change in texture of the material. It is also necessary to measure any ductile tearing prior to fracture. Again, this is discernible by a change in appearance of the fracture surface, although because all of the tests to date have failed through cleavage, this has be minimal, or zero in most of the cases.

\section{Experimental Jc distributions}

Cleavage fracture toughness data in terms of $J_{c}$-values were determined from the estimation procedure based on $\eta$-factors given in ASTM E1820 [20] using the experimentally measured plastic area under the $P-C M O D$ curve for each test specimen. Post-mortem observation of fracture surfaces reveal no ductile crack growth prior to cleavage fracture for all specimens. More- 
over, it was found that the average fatigue precrack ratio, based on a 9-point measurement technique, was set as $a / W=0.06$ for the very shallow crack specimens which is a little bit different than the desired initial ratio $(a / W=0.05)$. However, the use of specimen having very low crack size, $a / W=0.06$, can still be considered as novelty in the evaluation of the influence of stress triaxiality level on fracture toghness values.

The fracture toughness $J_{c}$-values are shown in Fig. 5 in graphical form and in table 1 in tabular form. The Weibull shape parameter $(\alpha)$ and the characteric toughness value $\left(J_{0}\right)$ are reported for each geometry in table 1 as well. The characteristic toughness $J_{0}$-value corresponds to a probability of failure equal to $P_{f}=63.2 \%$. It is worth to remember that a shape parameter $(\alpha)$ equal to 2 characterizes well the dispersion on toughness values due to cleavage fracture under small scale conditions at the fracture process zone.

A two-parameter Weibull distribution has been fitted to the experimental data to obtain $\alpha$ and $J_{0}$ by adopting the maximum likelihood method. The $95 \%$ confidence intervals for $\alpha$ and $J_{0}$ are as follow:

- Very shallow crack $(a / W=0.06): J_{0}=(97 ; 60.2)$ and $\alpha=$ $(4.6 ; 1.6)$

- Shallow crack $(a / W=0.2): J_{0}=(59 ; 37)$ and $\alpha=(4.6$; 1.6)

- Deep crack $(a / W=0.4): J_{0}=(51.5 ; 34.8)$ and $\alpha=(6.1$; 1.9)

TABLE 1. Summary of $J_{c}$-values obtained for each tested 3PB specimen at $T=-140^{\circ} \mathrm{C}$.

\begin{tabular}{|c|c|c|c|c|c|c|c|c|c|c|c|c|c|}
\hline \multirow{2}{*}{$\begin{array}{l}W=2 B \\
W=1 \text { in }\end{array}$} & \multirow{2}{*}{\begin{tabular}{|l|}
$\mathrm{S}=4 \mathrm{~W}$ \\
$J_{\mathrm{c}}[\mathrm{MPa}-\mathrm{mm}]$ \\
\end{tabular}} & \multicolumn{10}{|c|}{ Specimen Number \# } & \multirow{2}{*}{\begin{tabular}{|c|} 
Scale \\
$J_{0}$ \\
\end{tabular}} & \multirow{2}{*}{$\begin{array}{r}\text { Shape } \\
\alpha\end{array}$} \\
\hline & & 1 & 2 & 3 & 4 & 5 & 6 & 7 & 8 & 9 & 10 & & \\
\hline \multirow{3}{*}{$a / w$} & 0,06 & 18,1 & 25,4 & 49,5 & 64,4 & 108,7 & 81,1 & 89,4 & 68,8 & 96,0 & 78,8 & 80 & 2,71 \\
\hline & 0,2 & 55,0 & 60,7 & 72,2 & 24,2 & 45,8 & 35,1 & 37,1 & 46,2 & 25,4 & 16,2 & 48 & 2,75 \\
\hline & 0,4 & 46,1 & 40,5 & 47,9 & 45,9 & 56,1 & 9,3 & 39,2 & 28,4 & 31,1 & 25,0 & 42,4 & 3,4 \\
\hline
\end{tabular}

As can be seen in Fig. 5, there exist a quite large variation on $J_{c}$-values for each cracked specimen, specially for the very shallow crack which is more likely to develop plastic deformation at the crack tip (specimen having less constraint) during the loading.

\section{NUMERICAL PROCEDURES}

\section{Finite Element Models}

Nonlinear finite element analyses are described for 3D models of bending specimens matching the tested specimens. These configurations have standard geometry $(W=2 B$ with $S=4 W$ ) and no side-grooves as previously described.The material flow properties match those for the A533 Grade B steel tested at
$T=-140^{\circ} \mathrm{C}$ as depicted in Fig. 4. The tensile mechanical properties were inputted in a piecewise form. A piecewise-linear representation of the measured true stress- true strain curve must provide a better description of the experimental data as well as slightly improved computational efficiency for the numerical solutions than a power law representation.

Figure 6 shows the typical 3D finite element model utilized in the analyses of the deeply cracked $1 \mathrm{~T}$ SE(B) specimen. A conventional mesh configuration having a focused ring of elements surrounding the crack front is used with a small key-hole at the crack tip; the radius of the key-hole, $\rho_{0}$, is $5 \mu \mathrm{m}(0.05 \mathrm{~mm})$. Symmetry conditions enable analyses using one-quarter of the 3-D models with appropriate constraints imposed on the symmetry planes. The meshes have 30 variable thickness layers defined over the half-thickness $(B / 2)$; the thickest layer is defined at $Z=0$ with thinner layers defined near the free surface $(Z=B / 2)$ to accommodate strong $Z$ variations in the stress distribution. The quarter-symmetric, 3-D model for this specimen has approximately 57,400 nodes and 52,400 3-D elements. These finite element models are loaded by displacement increments imposed on a frictionless rigid cylinder using general contact available in Abaqus software.

The finite element code Abaqus [21] provides the numerical solutions for the detailed 3-D analyses reported here using a implicit integration scheme (Abaqus/Standard) to solve the equilibrium equations. The elastic-plastic constitutive model employed in the stationary crack analyses reported here follows a flow theory with conventional Mises plasticity in large geometry change (LGC) setting. Abaqus/Standard employs a domain integral method or virtual crack extension approach [22] for numerical evaluation of the $J$-integral to provide pointwise values of $J$ across the crack front at each loading level. The numerical reported $J$ values correspond to the midplane for each cracked specimen.

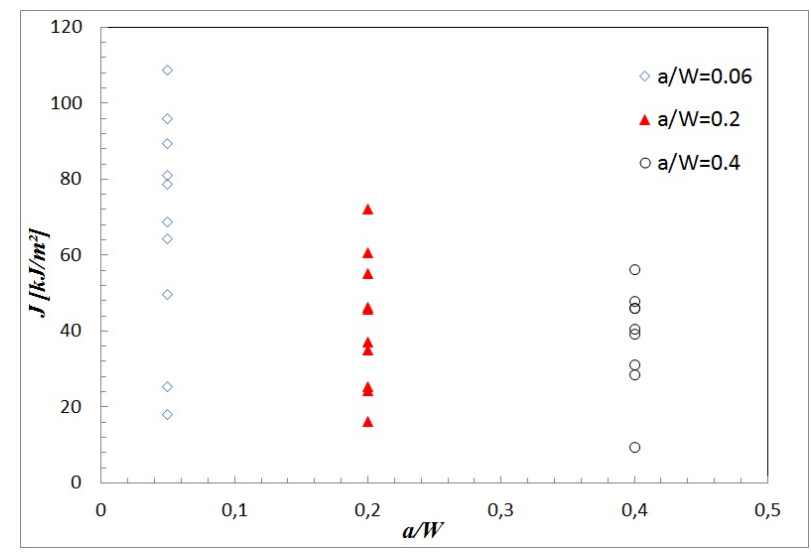

FIGURE 5. Fracture toughness test results for the 3PB specimens at $T=-140^{\circ} \mathrm{C}$. 


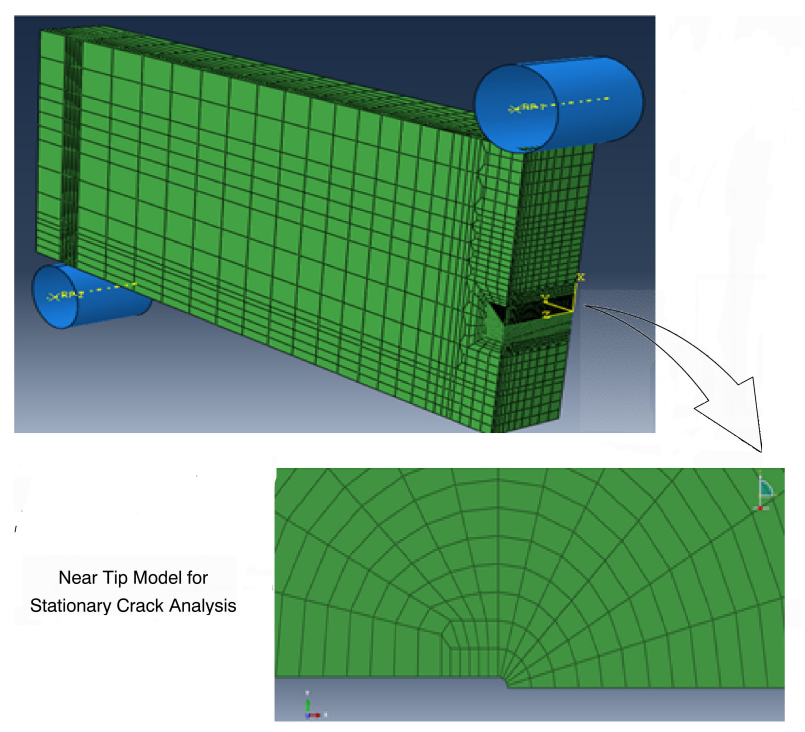

FIGURE 6. 3D finite element model for the 3PB SEB specimen with $a / W=0.4$.

To check the validity of the finite element models, it is made a direct comparison between measured and numerical $P$ $C M O D$-curves. Figure 7 compares the experimental and predicted load and crack mouth opening displacement curves for $\mathrm{SE}(\mathrm{B})$ specimens with very shallow crack ratio $a / W=0.06$ and deep crack ratio $a / W=0.4$. In general terms, the predictions of the $P-C M O D$-curves using the current finite element models correlate well with the experimental measurements. It must be noticed that the experimental results shown in Fig.7 correspond to three $\mathrm{SE}(\mathrm{B})$ specimens for both crack size ratios. It also has to be commented the good repeatability of the experimental results coming from different specimens, all $P-C M O D$ trajectories have similar slope at the beginning of loading and the followe path in the nonlinear portion is very close between all specimens.

\section{CLEAVAGE FRACTURE PREDICTIONS}

\section{Calibration of Weibull Parameters}

Numerical simulation of brittle fracture in 3PB specimens described here begins with calibration of the standard Weibull model parameters, $m_{0}, \sigma_{u}$, for the steel employed in this study. The Weibull shape parameter $m$ and scale parameter $\sigma_{u}$ define the key parameters for studying the fracture toughness variations due to geometry effects using the standard Weibull approach. The measured $J_{c}$-values, obtained from two sets of SE(B) specimens, having different constraint levels, are employed to calibrate these parameters. Within the present context, finite element analyses are conducted to calibrate the Weibull parameters which scale the experimentally measured characteristic toughness values between the high constraint fracture specimen $(a / W=0.4)$ and the very low contraint specimen $(a / W=0.06)$.

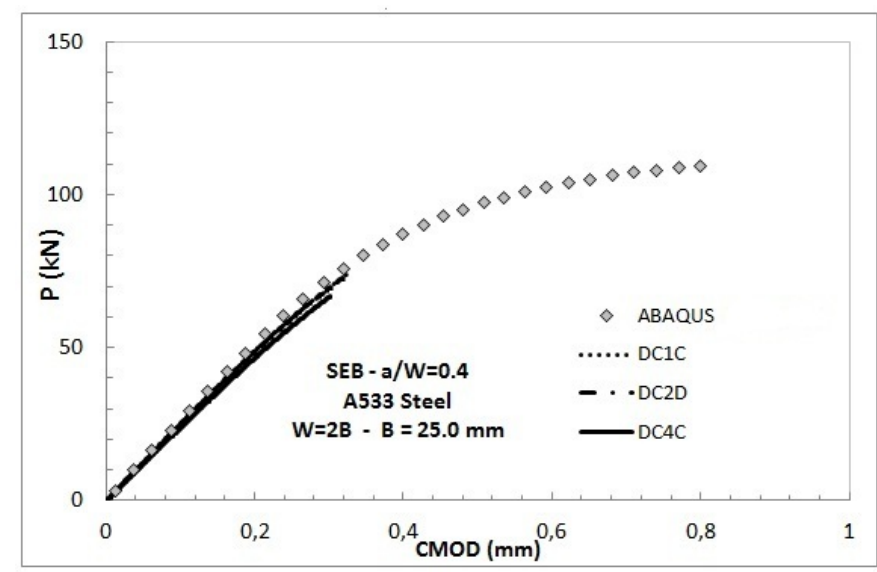

(a)

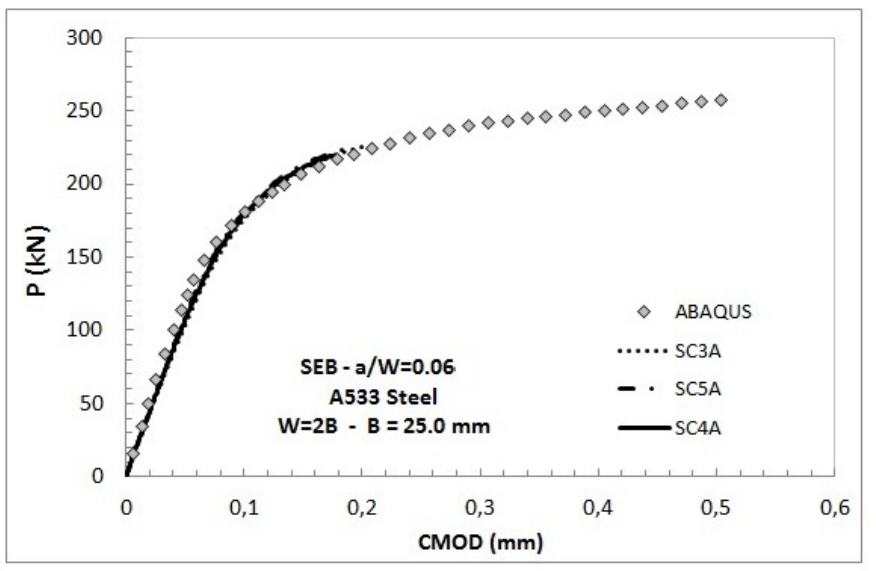

(b)

FIGURE 7. Comparison between experimental results and numerical predictions of $P$-CMOD curves for : (a) $\mathrm{SE}(\mathrm{B})$ geometry with a/W = 0.4 ; (b) $\mathrm{SE}(\mathrm{B})$ geometry with a/W $=006$.

To help the calibration process it is good idea to make a plot showing the variation of $J_{0}$-values for both geometries using different values for $\sigma_{w}$ as possible critical driving force at the crack tip. This is the so-called constraint correlation plot. Figure 8 displays the constraint correlation for varying values of Weibull shape parameter. For a curve with fixed $m_{0}$, each point gives a couple of possible characterictic toughness value for each cracked specimen having the same Weibull stress. Each point $\left[J_{a / W=0.06}, J_{a / W=0.4}\right]$ represents same probability of failure. A line having a slope equal to $45^{\circ}$ has been include as reference.

Figure 9 provides the variation of the Weibull stress $\sigma_{w^{-}}$ 
values with $J$-integral for the $\mathrm{SE}(\mathrm{B})$ fracture specimen with different $a / W$-ratios. The trends are similar as previous work reported in $[11,12]$. A nonlinear variation of $\sigma_{w}$ can be seen with increasing levels of remote loading, measured by the $J$-integral.

The calibration process has been performed using an inhouse Python routine. The fracture process (FPZ) zone is defined by the region where the maximum principal stresses are equal or higher than the yield stress of the material at the tested temperature. This condition is identified as $\lambda=1$. Similar computations were made using the von Mises stresses as the criteria to define the FPZ, resulting in similar results. The calibrated Weibull parameters, that match the experimental characteristic values $J_{0}$ for two different $\mathrm{SE}(\mathrm{B})$ specimens with different crack size, are $m_{0}=8.65$ and $\sigma_{u}=2297.2 \mathrm{MPa}$.

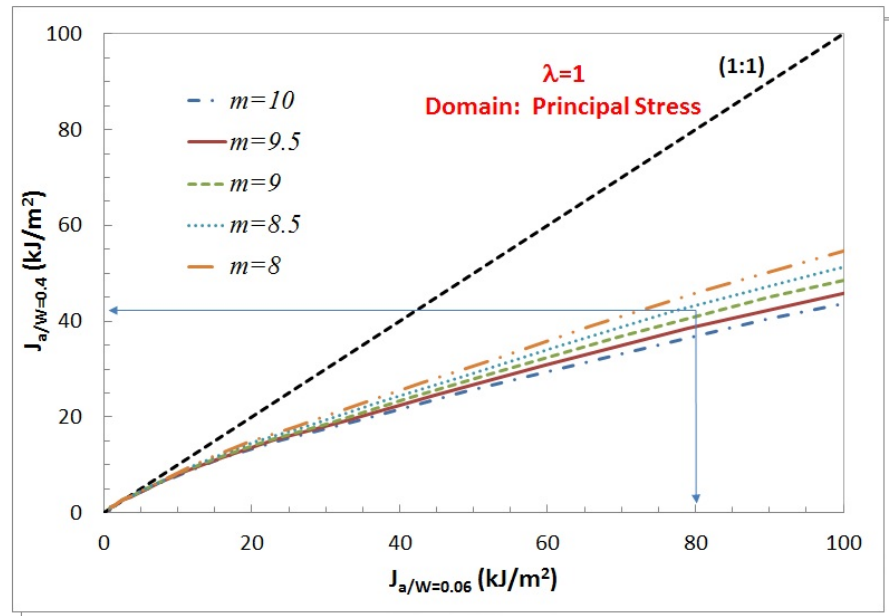

FIGURE 8. Constraint correlation of $J_{0}$-values for $\mathrm{SE}(\mathrm{B})$ specimens made of A533 steel at $T=-140^{\circ} \mathrm{C}$.

Using a constant Weibull stress $\sigma_{w}=2297.2 \mathrm{MPa}$ as a failure criteria, the Weibull trajectories predict $J_{0}=42.6 \mathrm{~kJ} / \mathrm{m}^{2}$ and $J_{0}$ $=80 \mathrm{~kJ} / \mathrm{m}^{2}$ for the high constraint and the very low constraint geometries, respectively. Thus, the toughness scaling procedure implemented in this work predicts the characteristic toughness $J_{0}$-value as $50 \mathrm{~kJ} / \mathrm{m}^{2}$ for the shallow crack specimen $(a / W=$ $0.2)$.

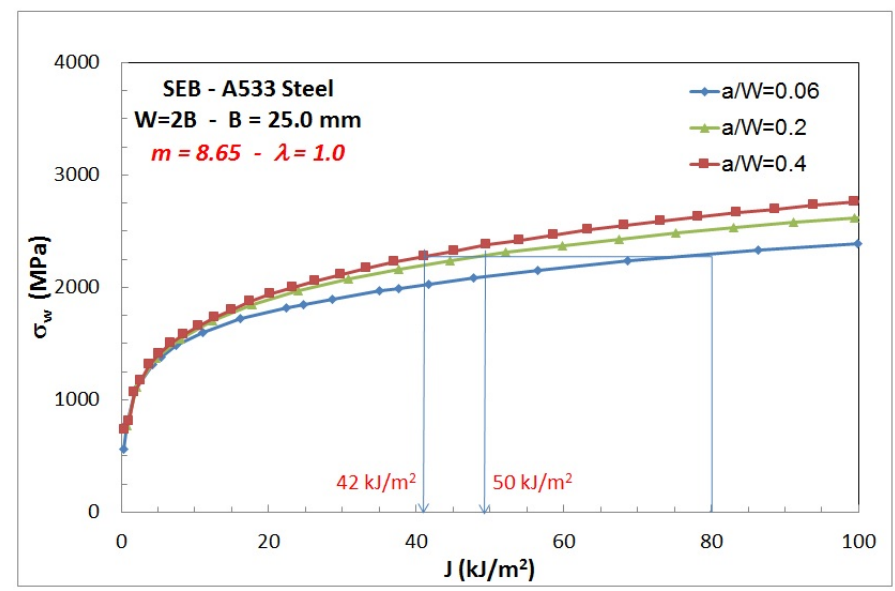

FIGURE 9. Evolution of Weibull stress $\sigma_{w}$-values for plane-side $1 \mathrm{~T}$ SE(B) specimens of A533 steel at $T=-140^{\circ} \mathrm{C}$ having different stress triaxialities.

It is worth to compare the evolution of $\sigma_{w}$ versus $J$-Integral for the geometries having $a / W=0.2$ and $a / W=0.4$. Even tough both geometries can be considered as components having different levels of crack tip constraint at the crack tip, the resulting trajectories of Weibull stress are very close to each other. Actually this is not an expected result. This mechanical response may be related to the very low temperature, $T=-140^{\circ} \mathrm{C}$, at which the tests were done. Thus, the material's ability to support plastic deformation is precluded by this very low tempertature.

Figures 10-11 provide the prediction of cumulative probability of failure $\left(F\left(\sigma_{w}\right)\right)$ as function of the $\sigma_{w}$-values. Figure 10 shows the best fit between experimental results and Weibull model wich results from the scaling toughness calibration methodology, explained in section. Figure 11 displays the prediction for the geometry that has not been used in the calibration. The continuous numerical function of $F\left(\sigma_{w}\right)$, in Figs. 10-11, has been plotted using the calibrated Weibull parameters $m_{0}=8.65$ and $\sigma_{u}=2297.2 \mathrm{MPa}$, as reported previously.

Altought a perfect match has not been obtained between the experimental distribution $\left(F\left(\sigma_{w}\right)\right)_{\text {Exp. }}$ and the calculated $\left(F\left(\sigma_{w}\right)\right)_{\text {Num. }}$. values obtained by the Weibull standard model, the agreement between both distribtuions is acceptable. Experimental and numerical cumulative distributiosn have less degree of matching for higher J-integral values. For the same probability of failure, the numerical prediction of critical $J_{c}$-value using the standard Weibull model is higher than the experimental measured toughness. 


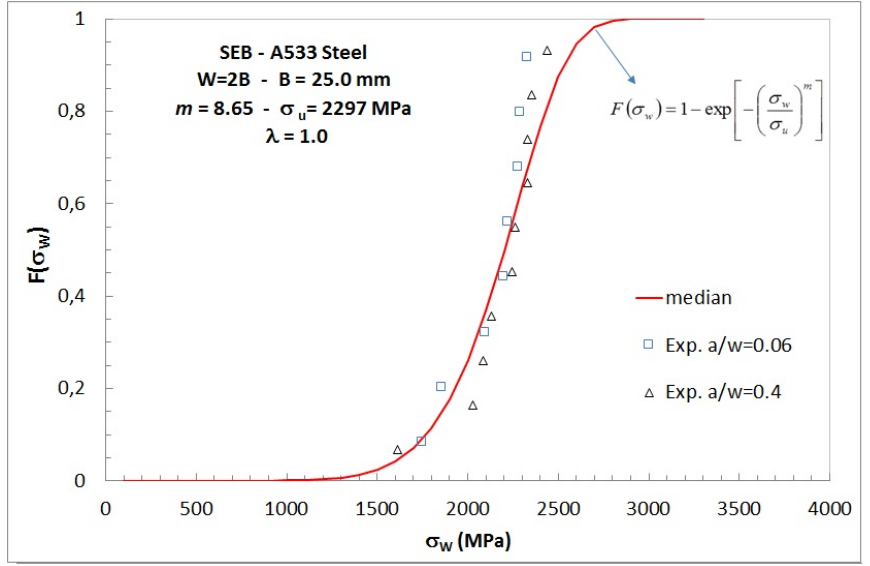

FIGURE 10. Comparison of Cumulative Weibull distributions obtained from numerical model and experimentally measured $J_{c}$-values for geometries with crack size ratios $a / W=0.4$ and $a / W=0.06$.

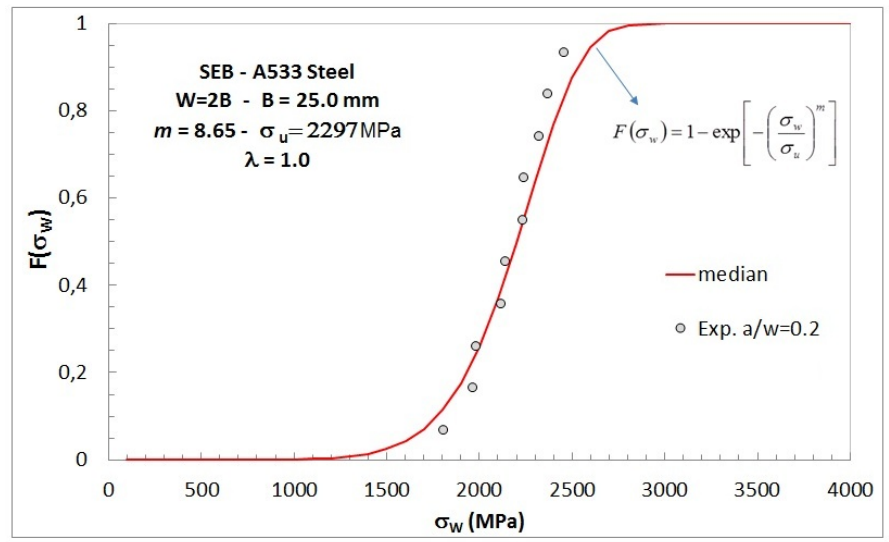

FIGURE 11. Comparison of Cumulative Weibull distributions obtained from numerical model and experimentally measured $J_{c}$-values for geometry with crack size ratio $a / W=0.2$.

\section{CONCLUDING REMARKS}

The extensive set of nonlinear 3D finite element analyses, for detailed plane-side models of SE(B) fracture specimens with varying crack sizes ratios described here, provide the basis to determine the effects of constraint loss on global values of fracture toughness for an A533 Gr. pressure vessel steel. The finite elements models were validated comparing the predicted and measured $P-C M O D$ curves.

As can be seen in Figures 9 - 11 the Standard Beremin Model is able to predict with a good degree of agreement the characteristic toughness values $J_{0}$ and the cumulative probability of failure $F\left(\sigma_{w}\right)$ to experimental results. The described model can take into account the effect of crack tip triaxiality on the material resistance to cleavage fracture. Also, the experimental results showed here, for the very shallow crack, can be considered as a novel group of fracture toughness data that will be available for the fracture community for further studies. Figures 9 clearly reveals a strong effect on levels of stress triaxiality at the crack tip zone as a result of the very low temperature $T=-140^{\circ} \mathrm{C}$ used to test the $\mathrm{SE}(\mathrm{B})$ specimens with initial crack size ratios $a / W=0.2$ and $a / W=0.4$. Consequently the $J_{0}$-value and evolution of $\sigma_{w}-J$ trajectories are very close for both specimens. Further work is in progress to test the ability of Beremin's standard Weibull model and Modified Weibull model to predict plastic strain effects and residual stresses on toughness values for the material described in this study.

\section{ACKNOWLEDGMENT}

This investigation is supported by the Royal Academy of Engineering throught the Newton Research Collaborative Program signed between University of Manchester (UK) and University of São Paulo (Brazil).

\section{REFERENCES}

[1] Rathbun, H. J., Odette, G. R., Yamamoto, T., and Lucas, G. E., 2006. "Influence of statistical and constraint loss size effects on cleavage fracture toughness in the transtion : Qa single variable experiment and database". Engineering Fracture Mechanics, 73, pp. 134-158.

[2] Savioli, R., and ruggieri, C., 2014. "Experimental study on the cleavage fracture behavior of an astm a285 grade c pressu vessel steel". ASME, 137(2), pp. 1-7.

[3] Beremin, F. M., 1983. "A local criterion for cleavage fracture of a nuclear pressure vessel steel". Metallurgical and Materials Transactions A, 14, pp. 2277-2287.

[4] Mudry, F., 1987. "A local approach to cleavage fracture". Nuclear Engineering and Design, 105, pp. 65-76.

[5] Pineau, A., 2006. "Development of the local approach to fracture over the past 25 years: Theory and applications". International Journal of Fracture, 138, pp. 139-166.

[6] McMahon, C. J., and Cohen, M., 1965. "Initiation of cleavage in polycrystalline iron". Acta Metallurgica, 13, pp. 591-604.

[7] Kaechele, L. E., and Tetelman, A. S., 1969. "A statistical investigation of microcrack formation". Acta Metallurgica, 17, pp. 475-475.

[8] Brindley, B. J., 1970. "The effect of dynamic strain-aging on the ductile fracture process in mild steel". Acta Metallurgica, 18, pp. 325-329.

[9] Lindley, T. C., Oates, G., and Richards, C. E., 1970. "A critical appraisal of carbide cracking mechanism in fer- 
ritic/carbide aggregates". Acta Metallurgica, 18, pp. 11271136.

[10] Gurland, J., 1972. "Observations on the fracture of cementite particles in a spheroidized 1.05 Acta Metallurgica, 20, pp. 735-741.

[11] Ruggieri, C., and Dodds, R. H., 2015. "An engineering methodology for constraint corrections of elastic-plastic fracture toughness-part i: A review on probabilistic models and exploration of plastic strain effects". Engineering Fracture Mechanics, 134, pp. 368-390.

[12] Ruggieri, C., Savioli, R. G., and Dodds, R. H., 2015. "An engineering methodology for constraint corrections of elastic-plastic fracture toughness-part ii: Effects of specimen geometru and plastic strain on cleavage fracture predictions". Engineering Fracture Mechanics, 146, pp. 185209.

[13] Wallin, K., Saario, T., and Törrönen, K., 1987. "Fracture of brittle particles in a ductile matrix". International Journal of Fracture, 32, pp. 201-209.

[14] Mann, N. R., Schager, R. E., and Singpurwalla, N. D., 1974. Methods for statistical analysis of reliability and life data.

[15] Wallin, K., and Laukkanen, A., 2008. "New developmets of the Wallin, Saario, Törrönen cleavage fracture model". Engineering Fracture Mechanics, 75, pp. 3367-3377.

[16] Ruggieri, C., and Dodds, R. H., 1996. "A transferability model for brittle fracture including constraint and ductile tearing effects: A probabilistic approach". International Journal of Fracture, 79, pp. 309-340.

[17] Gao, X., Ruggieri, C., and Dodds, R., 1998. "Calibration of weibull stress parameters using fracture toughness data". International Journal of Fracture, 92, pp. 175-200.

[18] Ruggieri, C., 2010. "An engineering methodology to assess effects of weld strength mismatch on cleavage fracture toughness using the weibull stress approachs". International Journal of Fracture, 163, pp. 231-252.

[19] , 2005. Fracture mechanics toughness tests.

[20] ASTM, 2008. "Standard test method for measurement of fracture toughness.". American Society for Testing and Materials, ASTM E1290.

[21] Smith, M., 2009. Abaqus/Standard User's Manual, Version 6.9.

[22] Shih, C., Moran, B., and Nakamura, T., 1986. "Energy release rate along a three-dimensional crack front ina thermally stressed body". International Journal of Fracture, 30(79-102). 